\title{
Formal Introduction to Fuzzy Implications
}

\author{
Adam Grabowski \\ Institute of Informatics \\ University of Białystok \\ Poland
}

\begin{abstract}
Summary. In the article we present in the Mizar system the catalogue of nine basic fuzzy implications, used especially in the theory of fuzzy sets. This work is a continuation of the development of fuzzy sets in Mizar; it could be used to give a variety of more general operations, and also it could be a good starting point towards the formalization of fuzzy logic (together with t-norms and t-conorms, formalized previously).
\end{abstract}

MSC: 03B52 68T37 03B35

Keywords: fuzzy implication; fuzzy set; fuzzy logic

MML identifier: FUZIMPL1, version: 8.1.06 5.44.1305

\section{INTRODUCTION}

As it is well known, the implication operator plays a crucial role in the classical two-valued logic. Based on this logical connective, we can define binary conjunction and disjunction, and also unary negation operator. In the field of fuzzy logic, the notions of t-norm and t-conorm are an abstraction of the classical conjunction and disjunction. Similarly, we can treat the notion of a fuzzy implication, as a generalization of a classical implication.

Fuzzy sets, a tool for modelling uncertainty, proposed by Zadeh [12], were formally introduced in Mizar in [10]. This approach is quite natural in the Mizar Mathematical Library [9, has rich continuation there [3] as it is significantly closer to set theory than another tool for doing so, namely rough sets by Pawlak [11, as recalled in the context of lattice theory in [5].

In order to cope with basic constructions present in the theory of fuzzy implications, we had to define a number of examples of binary connectives. It is 
especially important to have some, because taking into account the expressive power of registrations of clusters in the Mizar system and the role of attributes, most of theorems are stated in the form of the abovementioned registrations. Having prepared such formal background, properties can be calculated automatically via the mechanism of the type expansion.

In our formal approach, we follow closely the book [1].

A function $I:[0,1]^{2} \rightarrow[0,1]$ is called a fuzzy implication if it satisfies, for all $x, x_{1}, x_{2}, y_{1}, y_{2} \in[0,1]$, the following conditions:

$$
\begin{aligned}
& \text { if } x_{1} \leqslant x_{2}, \text { then } I\left(x_{1}, y\right) \geqslant I\left(x_{2}, y\right), \\
& \text { if } y_{1} \leqslant y_{2}, \text { then } I\left(x, y_{1}\right) \leqslant I\left(x, y_{2}\right), \\
& I(0,0)=1, \\
& I(1,1)=1, \\
& I(1,0)=0 .
\end{aligned}
$$

The functions satisfying equations (I3), (I4), and (I5) are called in our formalism, 00-dominant (Def. 3), 11-dominant (Def. 4), and 10-weak (Def. 5), respectively.

The mutual independence of the axioms was shown using $I_{-1}, I_{-2}, I_{-3}, I_{-4}$, $I_{-5}$ (see definitions Def. 9 - Def. 13 in the present paper) - each one violating exactly one among properties (I1) - (I5). Of course, these are not examples of fuzzy implications in the current setting, although Zadeh implication $I_{-1}$ is considered in the literature as multi-valued implication.

In the set of all fuzzy implications, denoted by $\mathcal{F} \mathcal{I}$, we have $I_{0}$ and $I_{1}$ as the least and the greatest elements (with the ordinary pointwise ordering of functions) for arbitrary $x, y \in[0,1]$ as

$$
\begin{aligned}
& I_{0}(x, y)= \begin{cases}1, & \text { if } x=0 \text { or } y=1 \\
0, & \text { otherwise }\end{cases} \\
& I_{1}(x, y)= \begin{cases}1, & \text { if } x<1 \text { or } y>0 \\
0, & \text { otherwise }\end{cases}
\end{aligned}
$$

Together with formal description of triangular norms and conorms (introduced in [2] and described in [7]) introducing fuzzy implications is the fundamental step towards defining fuzzy logic within the Mizar Mathematical Library. Both formal aproaches to the theory of rough and fuzzy sets could be compared in a more sophisticated way as initiated in [8]. Of course, the Mizar system is much more efficient in the logical reasoning than in calculations in the style of computer algebra systems (although in the field of rough sets it resulted in a number 
Table 0.1: Nine basic fuzzy implications ([1], p. 4)

\begin{tabular}{|c|c|c|c|}
\hline Name & Def. & \multirow{2}{*}{\multicolumn{2}{|c|}{$\begin{array}{l}\text { Defining formula } \\
I_{\mathrm{LK}}(x, y)=\min (1,1-x+y)\end{array}$}} \\
\hline Łukasiewicz & Def. 14 & & \\
\hline Gödel & Def. 16 & $I_{\mathrm{GD}}(x, y)=\{$ & $\begin{array}{ll}1, & \text { if } x \leqslant y \\
y, & \text { otherwise }\end{array}$ \\
\hline Reichenbach & Def. 17 & \multicolumn{2}{|c|}{$I_{\mathrm{RC}}(x, y)=1-x+x y$} \\
\hline Kleene-Dienes & Def. 18 & \multicolumn{2}{|c|}{$I_{\mathrm{KD}}(x, y)=\max (1-x, y)$} \\
\hline Goguen & Def. 19 & $I_{\mathrm{GG}}(x, y)=$ & $\begin{array}{ll}1, & \text { if } x \leqslant y \\
\frac{y}{x}, & \text { otherwise }\end{array}$ \\
\hline Rescher & Def. & $I_{\mathrm{RS}}(x, y)=$ & $\begin{array}{ll}1, & \text { if } x \leqslant y \\
0, & \text { if } x>y\end{array}$ \\
\hline Yager & Def. 21 & $I_{\mathrm{YG}}(x, y)=$ & $\begin{array}{ll}1, & \text { if } x=0 \text { and } y=0 \\
y^{x}, & \text { if } x>0 \text { or } y>0\end{array}$ \\
\hline Weber & Def. 22 & $I_{\mathrm{WB}}(x, y)=$ & $\begin{array}{ll}1, & \text { if } x<1 \\
y, & \text { if } x=1\end{array}$ \\
\hline Fodor & Def. 23 & $I_{\mathrm{FD}}(x, y)=$ & $\begin{array}{ll}1, & \text { if } x \leqslant y \\
\max (1-x, y), & \text { if } x>y\end{array}$ \\
\hline
\end{tabular}

of quite interesting observations [6]), hence formalizing fuzzy numbers [4] is less promising than the present one.

The main aim of the Mizar article was to introduce formally nine important examples of fuzzy implications (see Table 0.1).

\section{Preliminaries}

Let us consider elements $a, b$ of $[0,1]$. Now we state the propositions:

(1) $\max (b, \min (1-a, 1-b)) \in[0,1]$.

(2) $\min (1,1-a+b) \in[0,1]$.

(3) $1-a+(a \cdot b) \in[0,1]$.

(4) $\max (1-a, b) \in[0,1]$.

(5) If $a>0$ or $b>0$, then $b^{a} \in[0,1]$.

(6) If $a>b$, then $\frac{b}{a} \in[0,1]$. 


\section{Basic Attributes Defining Fuzzy Implications}

Let $f$ be a binary operation on $[0,1]$. We say that $f$ is antitone w.r.t. 1 st coordinate if and only if

(Def. 1) for every elements $x_{1}, x_{2}, y$ of $[0,1]$ such that $x_{1} \leqslant x_{2}$ holds $f\left(x_{1}, y\right) \geqslant$ $f\left(x_{2}, y\right)$.

We say that $f$ is isotone w.r.t. 2nd coordinate if and only if

(Def. 2) for every elements $x, y_{1}, y_{2}$ of $[0,1]$ such that $y_{1} \leqslant y_{2}$ holds $f\left(x, y_{1}\right) \leqslant$ $f\left(x, y_{2}\right)$.

We say that $f$ is 00 -dominant if and only if

(Def. 3) $f(0,0)=1$.

We say that $f$ is 11-dominant if and only if

(Def. 4) $f(1,1)=1$.

We say that $f$ is 10 -weak if and only if

(Def. 5) $f(1,0)=0$.

We say that $f$ is 01 -dominant if and only if

(Def. 6) $\quad f(0,1)=1$.

We say that $f$ has properties of a fuzzy implication if and only if

(Def. 7) $f$ is antitone w.r.t. 1st coordinate, isotone w.r.t. 2nd coordinate, 00dominant, 11-dominant, and 10-weak.

We say that $f$ has properties of a classical implication if and only if

(Def. 8) $f$ is 00-dominant, 01-dominant, 11-dominant, and 10-weak.

\section{Examples Showing Independence of Axioms}

The functor $I_{-1}$ yielding a binary operation on $[0,1]$ is defined by

(Def. 9) for every elements $x, y$ of $[0,1]$, it $(x, y)=\max (1-x, \min (x, y))$.

One can verify that $I_{-1}$ is isotone w.r.t. 2nd coordinate, 00-dominant, 11dominant, and 10-weak.

The functor $I_{-2}$ yielding a binary operation on $[0,1]$ is defined by

(Def. 10) for every elements $x, y$ of $[0,1]$, it $(x, y)=\max (y, \min (1-x, 1-y))$.

Let us note that $I_{-2}$ is antitone w.r.t. 1st coordinate, 00-dominant, 11dominant, and 10-weak.

The functor $I_{-3}$ yielding a binary operation on $[0,1]$ is defined by

(Def. 11) for every elements $x, y$ of $[0,1]$, if $y<1$, then $i t(x, y)=0$ and if $y=1$, then $i t(x, y)=1$. 
Let us observe that $I_{-3}$ is antitone w.r.t. 1st coordinate, isotone w.r.t. 2nd coordinate, non 00-dominant, 11-dominant, and 10-weak.

The functor $I_{-4}$ yielding a binary operation on $[0,1]$ is defined by

(Def. 12) for every elements $x, y$ of $[0,1]$, if $x=0$, then $i t(x, y)=1$ and if $x>0$, then $i t(x, y)=0$.

Observe that $I_{-4}$ is antitone w.r.t. 1st coordinate, isotone w.r.t. 2nd coordinate, 00-dominant, non 11-dominant, and 10-weak.

The functor $I_{-5}$ yielding a binary operation on $[0,1]$ is defined by

(Def. 13) for every elements $x, y$ of $[0,1], i t(x, y)=1$.

Observe that $I_{-5}$ is antitone w.r.t. 1 st coordinate, isotone w.r.t. 2nd coordinate, 00-dominant, 11-dominant, and non 10-weak.

\section{Catalogue of Fuzzy Implications}

The Łukasiewicz implication yielding a binary operation on $[0,1]$ is defined by

(Def. 14) for every elements $x, y$ of $[0,1]$, it $(x, y)=\min (1,1-x+y)$.

Note that the Eukasiewicz implication is antitone w.r.t. 1st coordinate, isotone w.r.t. 2nd coordinate, 00-dominant, 11-dominant, and 10-weak and there exists a binary operation on $[0,1]$ which has properties of a fuzzy implication and every binary operation on $[0,1]$ which has properties of a fuzzy implication is also antitone w.r.t. 1st coordinate, isotone w.r.t. 2nd coordinate, 00-dominant, 11-dominant, 10-weak.

Every binary operation on $[0,1]$ which is antitone w.r.t. 1st coordinate, isotone w.r.t. 2nd coordinate, 00-dominant, 01-dominant, 11-dominant, and 10-weak has also properties of a fuzzy implication and every binary operation on $[0,1]$ which has properties of a classical implication is also 00-dominant, 01-dominant, 11-dominant, 10-weak.

Every binary operation on $[0,1]$ which is 00-dominant, 01-dominant, 11dominant, and 10-weak has also properties of a classical implication and every binary operation on $[0,1]$ which has properties of a fuzzy implication has also properties of a classical implication.

A fuzzy implication is an antitone w.r.t. 1st coordinate, isotone w.r.t. 2nd coordinate, 00-dominant, 11-dominant, 10-weak binary operation on $[0,1]$. The functor $\mathcal{F} \mathcal{I}$ yielding a set is defined by the term

(Def. 15) the set of all $f$ where $f$ is a fuzzy implication.

The Gödel implication yielding a binary operation on $[0,1]$ is defined by

(Def. 16) for every elements $x, y$ of $[0,1]$, if $x \leqslant y$, then $i t(x, y)=1$ and if $x>y$, then $i t(x, y)=y$. 
Let us note that the Gödel implication is antitone w.r.t. 1st coordinate, isotone w.r.t. 2nd coordinate, 00-dominant, 11-dominant, and 10-weak.

The Reichenbach implication yielding a binary operation on $[0,1]$ is defined by

(Def. 17) for every elements $x, y$ of $[0,1]$, it $(x, y)=1-x+(x \cdot y)$.

Let us note that the Reichenbach implication is antitone w.r.t. 1st coordinate, isotone w.r.t. 2nd coordinate, 00-dominant, 11-dominant, and 10-weak.

The Kleene-Dienes implication yielding a binary operation on $[0,1]$ is defined by

(Def. 18) for every elements $x, y$ of $[0,1], i t(x, y)=\max (1-x, y)$.

Let us observe that the Kleene-Dienes implication is antitone w.r.t. 1st coordinate, isotone w.r.t. 2nd coordinate, 00-dominant, 11-dominant, and 10-weak.

The Goguen implication yielding a binary operation on $[0,1]$ is defined by

(Def. 19) for every elements $x, y$ of $[0,1]$, if $x \leqslant y$, then $i t(x, y)=1$ and if $x>y$, then it $(x, y)=\frac{y}{x}$.

One can verify that the Goguen implication is antitone w.r.t. 1st coordinate, isotone w.r.t. 2nd coordinate, 00-dominant, 11-dominant, and 10-weak.

The Rescher implication yielding a binary operation on $[0,1]$ is defined by

(Def. 20) for every elements $x, y$ of $[0,1]$, if $x \leqslant y$, then it $(x, y)=1$ and if $x>y$, then it $(x, y)=0$.

Let us note that the Rescher implication is antitone w.r.t. 1st coordinate, isotone w.r.t. 2nd coordinate, 00-dominant, 11-dominant, and 10-weak.

The Yager implication yielding a binary operation on $[0,1]$ is defined by

(Def. 21) for every elements $x, y$ of $[0,1]$, if $x=y=0$, then $i t(x, y)=1$ and if $x>0$ or $y>0$, then it $(x, y)=y^{x}$.

One can check that the Yager implication is antitone w.r.t. 1st coordinate, isotone w.r.t. 2nd coordinate, 00-dominant, 11-dominant, and 10-weak.

The Weber implication yielding a binary operation on $[0,1]$ is defined by

(Def. 22) for every elements $x, y$ of $[0,1]$, if $x<1$, then it $(x, y)=1$ and if $x=1$, then $i t(x, y)=y$.

Let us note that the Weber implication is antitone w.r.t. 1st coordinate, isotone w.r.t. 2nd coordinate, 00-dominant, 11-dominant, and 10-weak.

The Fodor implication yielding a binary operation on $[0,1]$ is defined by

(Def. 23) for every elements $x, y$ of $[0,1]$, if $x \leqslant y$, then it $(x, y)=1$ and if $x>y$, then it $(x, y)=\max (1-x, y)$.

One can check that the Fodor implication is antitone w.r.t. 1st coordinate, isotone w.r.t. 2nd coordinate, 00-dominant, 11-dominant, and 10-weak. 


\section{BOUNDARY FUZZY IMPLICATIONS}

The functor $I_{0}$ yielding a binary operation on $[0,1]$ is defined by

(Def. 24) for every elements $x, y$ of $[0,1]$, if $x=0$ or $y=1$, then $i t(x, y)=1$ and if $x>0$ and $y<1$, then it $(x, y)=0$.

One can verify that $I_{0}$ is antitone w.r.t. 1 st coordinate, isotone w.r.t. 2 nd coordinate, 00-dominant, 11-dominant, and 10-weak.

The functor $I_{1}$ yielding a binary operation on $[0,1]$ is defined by

(Def. 25) for every elements $x, y$ of $[0,1]$, if $x<1$ or $y>0$, then $i t(x, y)=1$ and if $x=1$ and $y=0$, then it $(x, y)=0$.

One can verify that $I_{1}$ is antitone w.r.t. 1 st coordinate, isotone w.r.t. 2 nd coordinate, 00-dominant, 11-dominant, and 10-weak.

Let $f$ be a binary operation on $[0,1]$. We say that $f$ satisfies (LB) if and only if

(Def. 26) for every element $y$ of $[0,1], f(0, y)=1$.

We say that $f$ satisfies (RB) if and only if

(Def. 27) for every element $x$ of $[0,1], f(x, 1)=1$.

Now we state the propositions:

(7) Let us consider a fuzzy implication $I$, and an element $y$ of $[0,1]$. Then $I(0, y)=1$.

(8) Let us consider a fuzzy implication $I$, and an element $x$ of $[0,1]$. Then $I(x, 1)=1$.

Observe that every fuzzy implication satisfies (LB) and (RB).

Let us consider a fuzzy implication $I$. Now we state the propositions:

(9) $I_{0} \leqslant I$. The theorem is a consequence of (7) and (8).

(10) $I \leqslant I_{1}$.

\section{REFERENCES}

[1] Michał Baczyński and Balasubramaniam Jayaram. Fuzzy Implications. Springer Publishing Company, Incorporated, 2008. doi:10.1007/978-3-540-69082-5.

[2] Adam Grabowski. Basic formal properties of triangular norms and conorms. Formalized Mathematics, 25(2):93-100, 2017. doi 10.1515/forma-2017-0009.

[3] Adam Grabowski. The formal construction of fuzzy numbers. Formalized Mathematics, 22(4):321-327, 2014. doi 10.2478/forma-2014-0032.

[4] Adam Grabowski. On the computer certification of fuzzy numbers. In M. Ganzha, L. Maciaszek, and M. Paprzycki, editors, 2013 Federated Conference on Computer Science and Information Systems (FedCSIS), Federated Conference on Computer Science and Information Systems, pages 51-54, 2013.

[5] Adam Grabowski. Lattice theory for rough sets - a case study with Mizar. Fundamenta Informaticae, 147(2-3):223-240, 2016. doi 10.3233/FI-2016-1406 
[6] Adam Grabowski and Magdalena Jastrzębska. Rough set theory from a math-assistant perspective. In Rough Sets and Intelligent Systems Paradigms, International Conference, RSEISP 200\%, Warsaw, Poland, June 28-30, 200\%, Proceedings, pages 152-161, 2007. doi:10.1007/978-3-540-73451-2_17.

[7] Adam Grabowski and Takashi Mitsuishi. Extending Formal Fuzzy Sets with Triangular Norms and Conorms, volume 642: Advances in Intelligent Systems and Computinq, pages 176-187. Springer International Publishing, Cham, 2018. doi 10.1007/978-3-319-668246_16.

[8] Adam Grabowski and Takashi Mitsuishi. Initial comparison of formal approaches to fuzzy and rough sets. In Leszek Rutkowski, Marcin Korytkowski, Ratal Scherer, Ryszard Tadeusiewicz, Lotfi A. Zadeh, and Jacek M. Zurada, editors, Artificial Intelligence and Soft Computing - 14th International Conference, ICAISC 2015, Zakopane, Poland, June 14-18, 2015, Proceedings, Part I, volume 9119 of Lecture Notes in Computer Science, pages 160-171. Springer, 2015. doi:10.1007/978-3-319-19324-3_15

[9] Adam Grabowski, Artur Korniłowicz, and Adam Naumowicz. Four decades of Mizar. Journal of Automated Reasoning, 55(3):191-198, 2015. doi:10.1007/s10817-015-9345-1

[10] Takashi Mitsuishi, Noboru Endou, and Yasunari Shidama. The concept of fuzzy set and membership function and basic properties of fuzzy set operation Formalızed Mathematıcs, 9(2):351-356, 2001.

[11] Zdzisław Pawlak. Rough sets. International Journal of Parallel Programming, 11:341-356, 1982. doi:10.1007/BF01001956

[12] Lotfi Zadeh. Fuzzy sets. Information and Control, 8(3):338-353, 1965.

Received September 3, 2017

The English version of this volume of Formalized Mathematics was financed under agreement 548/P-DUN/2016 with the funds from the Polish Minister of Science and Higher Education for the dissemination of science. 